\title{
Improved xylose and arabinose utilization by an industrial recombinant Saccharomyces cerevisiae strain using evolutionary engineering
}

\author{
Rosa Garcia Sanchez1 , Kaisa Karhumaa1,2, César Fonseca3,4, Violeta Sànchez Nogué1, João RM Almeida1,5, \\ Christer U Larsson'1, Oskar Bengtsson1,6, Maurizio Bettiga', Bärbel Hahn-Hägerdal'1 and Marie F Gorwa-Grauslund*1
}

\begin{abstract}
Background: Cost-effective fermentation of lignocellulosic hydrolysate to ethanol by Saccharomyces cerevisiae requires efficient mixed sugar utilization. Notably, the rate and yield of xylose and arabinose co-fermentation to ethanol must be enhanced.

Results: Evolutionary engineering was used to improve the simultaneous conversion of xylose and arabinose to ethanol in a recombinant industrial Saccharomyces cerevisiae strain carrying the heterologous genes for xylose and arabinose utilization pathways integrated in the genome. The evolved strain TMB3130 displayed an increased consumption rate of xylose and arabinose under aerobic and anaerobic conditions. Improved anaerobic ethanol production was achieved at the expense of xylitol and glycerol but arabinose was almost stoichiometrically converted to arabitol. Further characterization of the strain indicated that the selection pressure during prolonged continuous culture in xylose and arabinose medium resulted in the improved transport of xylose and arabinose as well as increased levels of the enzymes from the introduced fungal xylose pathway. No mutation was found in any of the genes from the pentose converting pathways.

Conclusion: To the best of our knowledge, this is the first report that characterizes the molecular mechanisms for improved mixed-pentose utilization obtained by evolutionary engineering of a recombinant $\mathrm{S}$. cerevisiae strain. Increased transport of pentoses and increased activities of xylose converting enzymes contributed to the improved phenotype.
\end{abstract}

\section{Background}

Lignocellulosic feedstocks, in particular agricultural wastes and hardwoods, contain a large fraction of the pentose sugars D-xylose and L-arabinose [1]. Therefore, cost-efficient fermentation of lignocellulosic hydrolysates to ethanol requires a micro-organism that can ferment pentose sugars to ethanol with high yield and productivity. Saccharomyces cerevisiae is the conventional microorganism for ethanol production, but it cannot naturally ferment pentose sugars. Consequently, various metabolic engineering strategies have been employed to express both fungal and bacterial genes encoding either D-xylose or L-arabinose utilizing pathways in S. cerevisiae

* Correspondence: Marie-Francoise.Gorwa@tmb.lth.se

1 Department of Applied Microbiology, Lund University, PO Box 124, SE-22100 Lund, Sweden

Full list of author information is available at the end of the article (reviewed recently in, for example, $[2,3]$ ). Despite these efforts, the sole heterologous expression of these genes was not sufficient to enable efficient fermentation to ethanol and additional modifications were required to overcome limitations in the conversion of pentoses.

Using isogenic S. cerevisiae strain background, higher specific ethanol productivity from xylose was achieved in the strain expressing the xylose reductase (XR) and xylitol dehydrogenase $(\mathrm{XDH})$ pathway (Figure 1), whereas higher ethanol yield was obtained when expressing the fungal xylose isomerase pathway [4]. With arabinose, the first functional bacterial arabinose pathway (Figure 1) expressed in S. cerevisiae combined the Bacillus subtilis AraA and Escherichia coli AraB and AraD [5]. Later studies have used homologous genes from Lactobacillus plantarum [6] or B. licheniformis AraA [7] to improve 


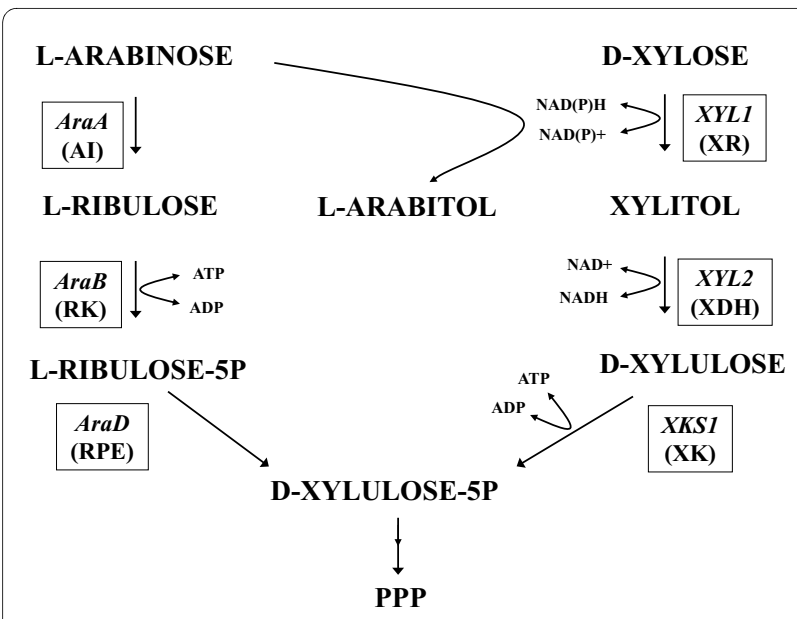

Figure $1 \mathrm{D}$-xylose and L-arabinose utilization pathways engineered in the Saccharomyces cerevisiae strains TMB3061, TMB3063 and TMB3130. Fungal D-xylose utilizing pathway: Pichia stipitis D-xylose reductase (XR), XYL1; P. stipitis D-xylitol dehydrogenase $(X D H), X Y L 2$; S. cerevisiae D-xylulose kinase (XK); XKS1. Bacterial L-arabinose utilizing pathway: Bacillus subtilis L-arabinose isomerase (AI), AraA; Escherichia coli L-ribulokinase (RK), AraB; E. coli L-ribulose-5-P 4 epimerase (RPE), AraD.

arabinose utilization. The fungal arabinose-utilization pathway has been also engineered in S. cerevisiae strains [8] enabling growth on arabinose.

Ethanol productivity would benefit from the design of stable $S$. cerevisiae strains that would co-consume xylose and arabinose, since both sugars are present in lignocellulosic feedstock. Co-expression of arabinose and xylose pathways in S. cerevisiae has been reported $[6,9,10]$. However, so far there is only one example of chromosomal integration of xylose and arabinose pathway genes in S. cerevisiae [10]. In these engineered industrial strains (named TMB3061 and TMB3063) that carry the Pichia stipitis xylose pathway combined with the B. subtilis - E. coli arabinose pathway (Figure 1), the consumption of arabinose and xylose was low and ethanolic fermentation was limited by extensive arabitol formation from XR enzyme [10] (Figure 1).

Evolutionary engineering (reviewed by [11]) complements rationally designed genetic engineering approaches. In the field of pentose fermentation, several examples of evolved $S$. cerevisiae strains that are improved for either xylose [12-15] or arabinose [5,6] fermentation have been reported. One more recent study describes the evolution of a S. cerevisiae strain towards both xylose and arabinose utilization by an haploid laboratory strain harbouring xylose and arabinose genes on multicopy plasmids [16].

In the present contribution, we report on an evolutionary engineering study to improve mixed sugar fermentation by the industrial polyploid $S$. cerevisiae strain
TMB3061 previously engineered with integrated genes for xylose and arabinose co-utilization [10]. Carbon-limited continuous culture with xylose and arabinose and increasing dilution rate was used to select for a population with improved growth on a mixture of xylose and arabinose sugars. One isolate, TMB3130, was further physiologically characterized.

\section{Results}

Evolutionary engineering for improved xylose and arabinose co-utilization

The industrial S. cerevisiae strain TMB3061 (Table 1) that overexpresses B. subtilis AraA, E. coli AraB and AraD, P. stipitis XYL1 and XYL2 and S. cerevisiae XKS1 [10] (Figure 1) was used to initiate the evolution in continuous culture under aerobic conditions using xylose and arabinose as limiting carbon sources. The feed initially contained $10 \mathrm{~g} \mathrm{~L}^{-1}$ xylose as the sole carbon source and it was changed to a mixture of $5 \mathrm{~g} \mathrm{~L}^{-1}$ xylose and $5 \mathrm{~g} \mathrm{~L}^{-1}$ arabinose after more than $400 \mathrm{~h}$, equivalent to 20 generations. The dilution rate was manually increased whenever a steady-state (assessed by stable cell concentration) was reached or decreased when washout was observed.

After approximately 65 generations, the dilution rate had been increased from 0.02 to $0.06 \mathrm{~h}^{-1}$ and the cell mass in the continuous culture reached an optical density (OD $620 \mathrm{~nm}$ ) higher than 20 as compared to initial values of 2-8 at the beginning of the experiment. A sample of the cell population after 70 generations was then chosen for further analysis.

\section{Isolation of the evolved strain TMB3130}

The population harvested from the continuous culture was sequentially grown in shake flask cultures containing first arabinose and then xylose in order to select for isolates that grew efficiently on both carbon sources. Cells from the shake flask culture on xylose were streaked on yeast peptone glucose (YPD) for isolation of single colonies. Each colony was re-streaked on yeast nitrogen base (YNB) plates containing xylose or arabinose as sole carbon source and 20 selected isolates able to grow efficiently on both pentoses were selected. Further analysis of 11 isolates was conducted in YNB liquid cultures where the specific growth rate $\left(\mu_{\max }\right)$ and the final biomass concentration (maximum OD $620 \mathrm{~nm}$ ) were compared to identify the best-growing isolates.

At least a twofold increase in maximum $\mathrm{OD}_{620 \mathrm{~nm}}$ was obtained for all isolates as compared to the parental strain TMB3061 when grown on xylose (data not shown). On the contrary, the ability to grow on arabinose appeared to be very divergent between the isolates, ranging from $\mu_{\max }$ 0.020 to 0.041 . While most isolates reached the stationary phase at an $\mathrm{OD}_{620 \mathrm{~nm}}$ between 1.2 and 2.2 in medium with 
Table 1: Description and aerobic growth on pentoses for the Saccharomyces cerevisiae strains used in the study

\begin{tabular}{|c|c|c|c|c|c|c|c|}
\hline \multirow[t]{2}{*}{ Strains } & \multirow[t]{2}{*}{ Relevant genotype/phenotype } & \multirow[t]{2}{*}{ Abbreviation } & \multicolumn{2}{|c|}{$\begin{array}{l}\text { Maximum specific } \\
\text { growth rate }\left(h^{-1}\right)\end{array}$} & \multicolumn{2}{|c|}{$\begin{array}{l}\text { Maximum } O_{620 \mathrm{~nm}} \\
\text { (average) }\end{array}$} & \multirow[t]{2}{*}{$\begin{array}{c}\text { Strain } \\
\text { reference }\end{array}$} \\
\hline & & & Xylose & Arabinose & Xylose & Arabinose & \\
\hline TMB3400 & $\begin{array}{l}\text { HIS3::ADH1p-XYL1-ADH1t, PGKp-XYL2-PGKt, } \\
\text { pPGK-XKS1-tPGK }\end{array}$ & $\mathrm{x}$ & $0.129 \pm 0.010$ & $0.002 \pm 0.000$ & 19.1 & 0.5 & [31] \\
\hline TMB3061 & $\begin{array}{l}\text { TMB3400, TRP1:: YIpAraB KanMX, } \\
\text { NTS2::HXT7p-AraA-CYC1t and } \\
\text { NTS2:: HXT7p-AraD-CYC1t, KanMX }\end{array}$ & $X+A$ & $0.016 \pm 0.000$ & $0.014 \pm 0.000$ & 11.1 & 5.1 & [10] \\
\hline TMB3063 & $\begin{array}{l}\text { TMB3061, additional copies of } \\
\text { NTS2::HXT7p-AraA-CYC1t }\end{array}$ & $X+A+A$ & $0.027 \pm 0.000$ & $0.033 \pm 0.000$ & 12.1 & 5.0 & [10] \\
\hline TMB3130 & TMB3061, evolved on xylose and arabinose & $X+A$ evolved & $0.054 \pm 0.003$ & $0.027 \pm 0.010$ & 23.3 & 21.5 & This study \\
\hline
\end{tabular}

arabinose, two isolates continued growing until an $\mathrm{OD}_{620}$ ${ }_{\mathrm{nm}}$ of about 16 . When these two isolates had been transferred several times to fresh arabinose medium, $\mu_{\max }$ reached $0.040 \mathrm{~h}^{-1}$ for both isolates indicating that $\mu_{\max }$ on arabinose had been increased threefold compared with the parental strain TMB3061 ( $\left.\mu_{\max } 0.014 \mathrm{~h}^{-1}\right)$ (Table 1).

One of the two isolates was chosen for further characterization and named TMB3130 (Table 1). Analytical polymerase chain reaction (PCR) of the overexpressed XKS1 confirmed that TMB3130 originated from the recombinant strain TMB3061.

\section{Aerobic growth on pentose sugars}

The parental strain, TMB3061, and the newly isolated TMB3130 were compared with respect to aerobic growth on xylose and arabinose. The comparison also included: (i) TMB3400 which only harbours the heterologous xylose utilizing pathway and is the parental strain of TMB3061; and (ii) TMB3063 that is based on TMB3061 but has additional copies of AraA (Table 1) [10]. All strains were pre-grown on glucose before determination of $\mu_{\max }$ on pentose sugars.

The evolved strain TMB3130 displayed an enhanced aerobic growth rate on xylose (three- to fourfold) and on arabinose (twofold) compared with its parental strain TMB3061 (Table 1). However, the $\mu_{\max }$ on xylose was still twofold lower than that of the xylose-utilizing TMB3400, the xylose-utilizing parental strain of TMB3061 [10] (Table 1). The lag-phase on pentoses of the two xylose and arabinose co-consuming strains was also longer than that of TMB3400 (Figure 2) and, thus, appeared to be related to the introduction of the arabinose-utilizing pathway. However, after adaptation, the novel isolate TMB3130 reached the highest final biomass concentration in both xylose containing-medium and in arabinosecontaining medium (Table 1; Figure 2).

\section{Anaerobic co-fermentation of glucose, xylose and arabinose}

Mixed sugar fermentation with the evolved isolate TMB3130 was compared with the xylose-consuming strain TMB3400 and with the xylose- and arabinose coconsuming strain TMB3063 (Table 2). Glucose was included in the medium together with xylose and arabinose to allow anaerobic growth at the start of the fermentation.

Xylose and arabinose consumption rates were calculated during the pentose phase after exhaustion of glucose. The specific consumption rates of xylose and arabinose (Table 2) were the highest for the evolved strain TMB3130 and similar for the two pentose sugars (0.9$\left.0.10 \mathrm{~g}(\mathrm{~g} \text { cell h})^{-1}\right)$. For the two other strains, the xylose consumption rate was higher than the arabinose consumption rate. Almost all xylose and arabinose were consumed by the evolved strain TMB3130, while 46\% of xylose and $67 \%$ of arabinose were left in the medium by TMB3063. TMB3400 consumed almost all xylose but no arabinose, in accordance with its genetic make-up (Tables 1 and 2; Figure 3).

At the metabolite level, the ethanol yield was the highest in the evolved strain ( $0.29 \mathrm{~g}$ ethanol. g consumed xylose $^{-1}$; Table 2). Almost all arabinose was converted to arabitol in the three strains (Table 2). TMB3400 (X) had 


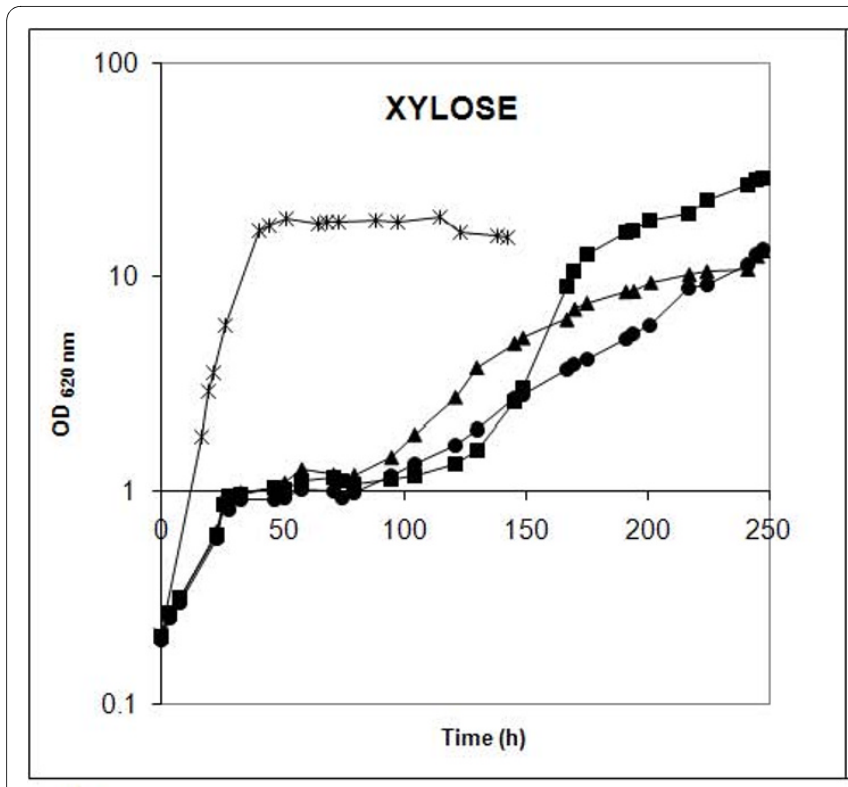

A

Figure 2 Aerobic growth of Saccharomyces cerevisiae strains on pentose sugars. (A) Yeast nitrogen base (YNB) medium with $50 \mathrm{~g} \mathrm{~L}^{-1} \mathrm{xylose}$ and (B) YNB medium with $50 \mathrm{~g} \mathrm{~L}^{-1}$ arabinose. Cells were pre-grown on YNB medium with $20 \mathrm{~g} \mathrm{~L}^{-1}$ glucose. TMB3400 (X) (asterisk); TMB3061 (X+A) (filled circle); TMB3063 (X+A+A) (filled triangle); and TMB3130 (X+A evolved) (filled square).

the highest xylitol yield 0.42 ( $\mathrm{g}$ xylitol. $\mathrm{g}$ xylose consumed $)^{-1}$ while TMB3063 $(\mathrm{X}+\mathrm{A}+\mathrm{A})$ and TMB3130 $(\mathrm{X}+\mathrm{A}$ evolved) had xylitol yields of 0.21 and 0.31 , respectively. The glycerol yield from consumed xylose was higher for TMB3400 (0.09 g glycerol. g consumed xylose $\left.\mathrm{e}^{-1}\right)$ and the lowest for TMB 3063 and TMB3130 (0.03 and $0.04 \mathrm{~g}$ glycerol. g consumed xylose ${ }^{-1}$, respectively; Table 2). In contrast, the acetate yield from consumed xylose was lower for TMB3400 (0.02 g acetate. $g$ consumed xylose $\left.\mathrm{e}^{-1}\right)$ and higher for TMB3063 (0.10 g acetate. g consumed xylose $\left.{ }^{-1}\right)$ and TMB3130 (0.08 g acetate. g consumed xylose ${ }^{-1}$; Table 2).

Enzymatic activities of the xylose and arabinose pathways $\mathrm{XR}$ and XDH activities of glucose-grown strain TMB3130 $(\mathrm{X}+\mathrm{A}$ evolved) were compared to those previously obtained with glucose grown cells for the parental strain TMB3061 (X+A) and strains TMB3400 (X) and TMB3063 $(\mathrm{X}+\mathrm{A}+\mathrm{A})[10]$. XR and XDH activities had significantly increased in the evolved isolate TMB3130, fiveto eightfold for XR and two- to 3.5-fold for XDH as compared to the activities measured in the other three strains (Table 3).

It was not possible to measure enzymatic activities of the arabinose pathways due to lack of commercially available compounds for the assays.

\section{Sequencing of genes from the xylose and arabinose pathways}

The genes encoding the heterologous arabinose and xylose pathways in the strains TMB3061 and TMB3130

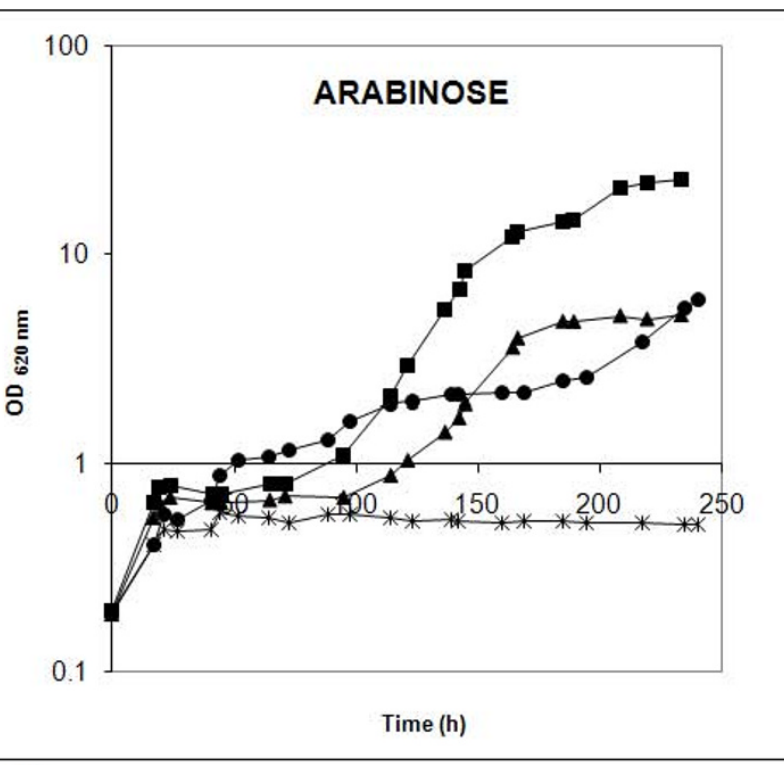

B were sequenced and no difference in nucleotide sequence was detected between the two strains. However, the $B$. subtilis AraA sequence of both TMB3130 and TMB3061 was found to contain nine point mutations compared with the original B. subtilis sequence found in GeneBank. Three of these mutations were non-silent, common for both strains and were positioned in aminoacids 201 (Phe T Leu), 366 (Ser T Pro) and 378 (Leu T Phe). Also the $E$. coli AraB sequence of TMB3061 and TMB3130 had three point mutations compared with the original E. coli sequence of which one was non-silent in position 121 (Asp T Asn) [5]. In both strains the P. stipitis XYL2 gene contained four silent point mutations.

\section{Assessment of transport kinetics}

S. cerevisiae strains TMB3400, TMB3061 and TMB3130 were characterized in terms of initial $\mathrm{D}-\left[\mathrm{U}-{ }^{14} \mathrm{C}\right] \mathrm{xylose}$ uptake rates in D-glucose- and D-xylose-grown cells. The three strains presented saturation kinetics with the presence of multiple transport systems for D-xylose, as denoted by the non-linear Eadie-Hofstee plots (Figure 4). When grown in D-glucose, the overall $K_{\mathrm{m}}$ for D-xylose uptake was very high (estimated above $300 \mathrm{mM}$ ) and similar in the three strains (data not shown). Although $K_{\mathrm{m}}$ values were higher than the maximal sugar concentration tested, the transport capacity for D-xylose at this concentration was estimated for comparative purposes: $33 \pm 6$

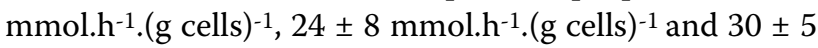
mmol.h $^{-1}$.(g cells) $)^{-1}$ for TMB3400 (X), TMB3061 (X+A) and TMB3130 (X+A evolved), respectively. 
Table 2: Anaerobic co-fermentation on $20 \mathrm{~g} \mathrm{~L}^{-1}$ glucose, $20 \mathrm{~g} \mathrm{~L}^{-1}$ xylose and $20 \mathrm{~g} \mathrm{~L}^{-1}$ arabinose on defined medium

\begin{tabular}{|c|c|c|c|c|c|c|c|c|c|}
\hline \multirow[t]{2}{*}{ Strains } & \multicolumn{2}{|c|}{ 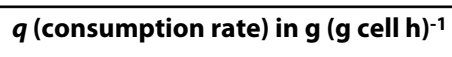 } & \multicolumn{2}{|c|}{ g sugar left at $120 \mathrm{~h}$} & \multirow{2}{*}{$\begin{array}{l}Y \text { arabitol (g arabitol. } g \\
\text { consumed arabinose }^{-1} \text { ) }\end{array}$} & \multicolumn{4}{|c|}{$Y\left(\right.$ g. $g$ consumed xylose $\left.^{-1}\right)$} \\
\hline & Xylose & Arabinose & Xylose & Arabinose & & Y xylitol & Y glycerol & Y acetate & Y ethanol \\
\hline TMB3400 & $0.07 \pm 0.00$ & $0.01 \pm 0.01$ & $0.23 \pm 0.06$ & $18.40 \pm 0.12$ & $0.94 \pm 0.02$ & $0.42 \pm 0.01$ & $0.09 \pm 0.01$ & $0.02 \pm 0.00$ & $0.22 \pm 0.01$ \\
\hline TMB3063 & $0.06 \pm 0.02$ & $0.05 \pm 0.02$ & $9.71 \pm 1.35$ & $13.97 \pm 1.59$ & $1.03 \pm 0.08$ & $0.21 \pm 0.01$ & $0.03 \pm 0.00$ & $0.10 \pm 0.09$ & $0.24 \pm 0.03$ \\
\hline TMB3130 & $0.09 \pm 0.02$ & $0.10 \pm 0.04$ & $2.11 \pm 0.22$ & $1.61 \pm 2.28$ & $0.95 \pm 0.05$ & $0.31 \pm 0.01$ & $0.04 \pm 0.00$ & $0.08 \pm 0.02$ & $0.29 \pm 0.12$ \\
\hline
\end{tabular}

The consumption rates of xylose and arabinose as well as the yields of arabitol, xylitol, glycerol, ethanol and acetate were calculated from the pentose phase after glucose depletion. The cell dry weight at the end of fermentation was used for the calculation of the specific consumption rates. TMB3400 only harbours the heterologous xylose-utilizing pathway; TMB3063 is a derivative from TMB3061 (that contains the xylose and arabinose pathways) with extra-copies of AraA gene; TMB3130 is evolved from TMB3061. 

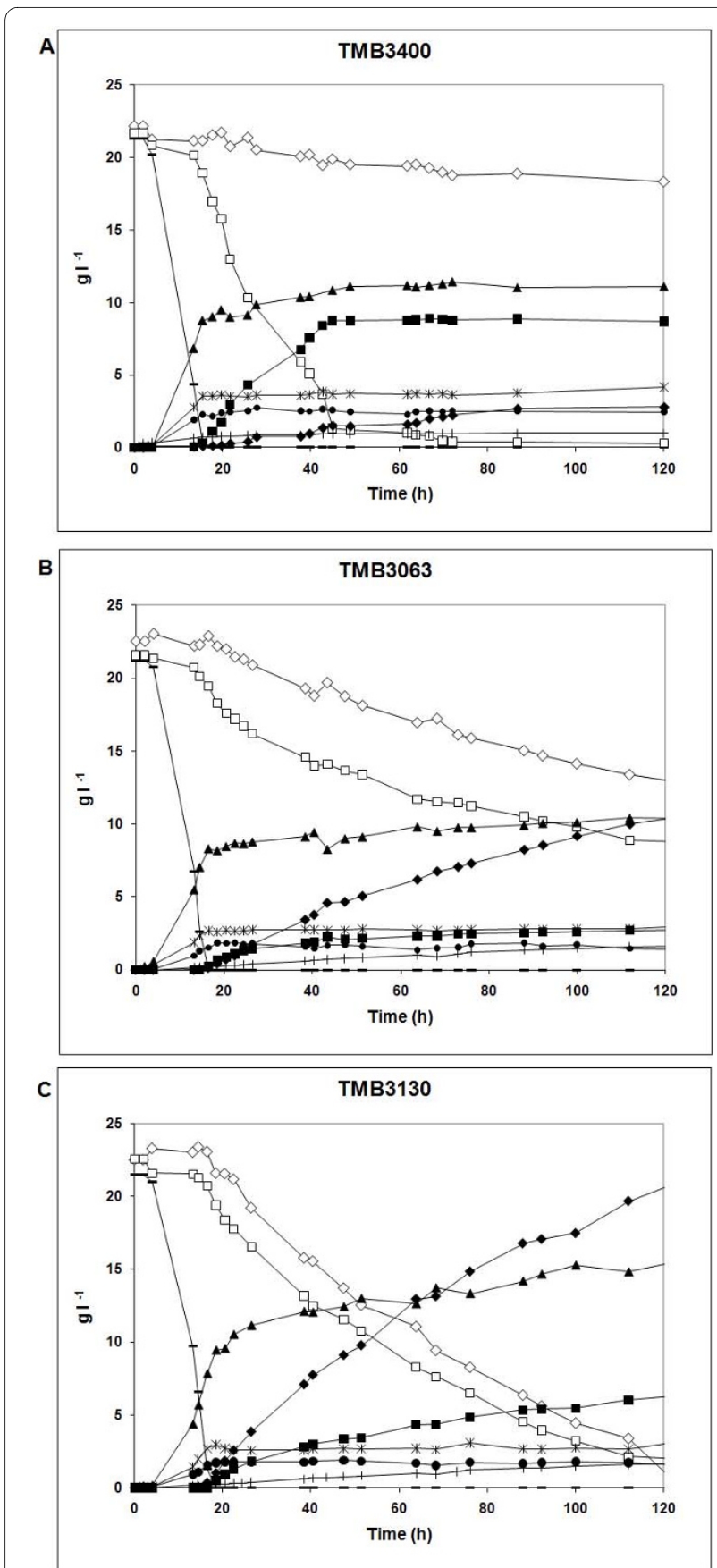

Figure 3 Anaerobic co-fermentation on defined medium with 20 $\mathrm{g} \mathrm{L}^{-1}$ glucose, $20 \mathrm{~g} \mathrm{~L}^{-1}$ xylose and $20 \mathrm{~g} \mathrm{~L}^{-1}$ arabinose. Cells for the inocula were pre-grown on defined medium with $20 \mathrm{~g} \mathrm{~L}^{-1}$ glucose. The strains used were Saccharomyces cerevisiae (A) TMB3400, (B) TMB3063 and (C) TMB3130. Symbols: glucose (minus symbol), xylose (open square), arabinose (open diamond), xylitol (filled square), arabitol (filled diamond), ethanol (filled triangle), biomass (filled circle), glycerol (asterisk), acetate (plus symbol).

When grown in D-xylose, TMB3400 showed similar kinetic parameters for xylose uptake as when grown in Dglucose (Figure 4). In contrast, TMB3061 and TMB3130 showed higher D-xylose uptake rates (1.5- to twofold) for
Table 3: Xylose reductase (XR) and xylitol dehydrogenase (XDH) activity (U.mg protein ${ }^{-1}$ ) of crude protein extracts from cells grown in defined medium with $20 \mathrm{~g} \mathrm{~L}^{-1}$ glucose

\begin{tabular}{ccc}
\hline Strain & $\begin{array}{c}\text { XR activity } \\
\mathbf{U} \mathbf{~ m g}^{-1} \text { protein }\end{array}$ & $\begin{array}{c}\text { XDH activity } \\
\mathbf{U ~ m g}^{-1} \text { protein }\end{array}$ \\
\hline TMB 3400 & $0.09 \pm 0.00\left(^{*}\right)$ & $0.59 \pm 0.08\left(^{*}\right)$ \\
\hline TMB3061 & $0.13 \pm 0.01\left(^{*}\right)$ & $0.52 \pm 0.03\left(^{*}\right)$ \\
\hline TMB 3063 & $0.10 \pm 0.01\left(^{*}\right)$ & $0.81 \pm 0.08\left(^{*}\right)$ \\
\hline TMB 3130 & $0.72 \pm 0.06$ & $1.85 \pm 0.61$ \\
\hline
\end{tabular}

${ }^{*}$ Taken from [10]

TMB3400 only harbours the heterologous xylose-utilizing pathway; TMB3063 is a derivative from TMB3061 (that contains the xylose and arabinose pathways) with extra-copies of AraA gene; TMB3130 is evolved from TMB3061.

each tested concentration, which apparently resulted from a lower overall $K_{\mathrm{m}}$ for D-xylose (estimated around $250 \mathrm{mM}$ both for TMB 3061 and TMB 3130 (Figure 4)).

When comparing strains, the transport capacity for xylose in the strains pre-grown in D-glucose (g) or Dxylose ( $\mathrm{x}$ ) was ranked as follows: TMB3061 (g) < TMB3130 (g) < TMB3400 (g) TMB3400 (x) < TMB3061 $(\mathrm{x})<\mathrm{TMB} 3130(\mathrm{x})$. Xylose transport was around 1.2- to 1.5-fold higher in TMB 3130 as compared to its parental TMB 3061.

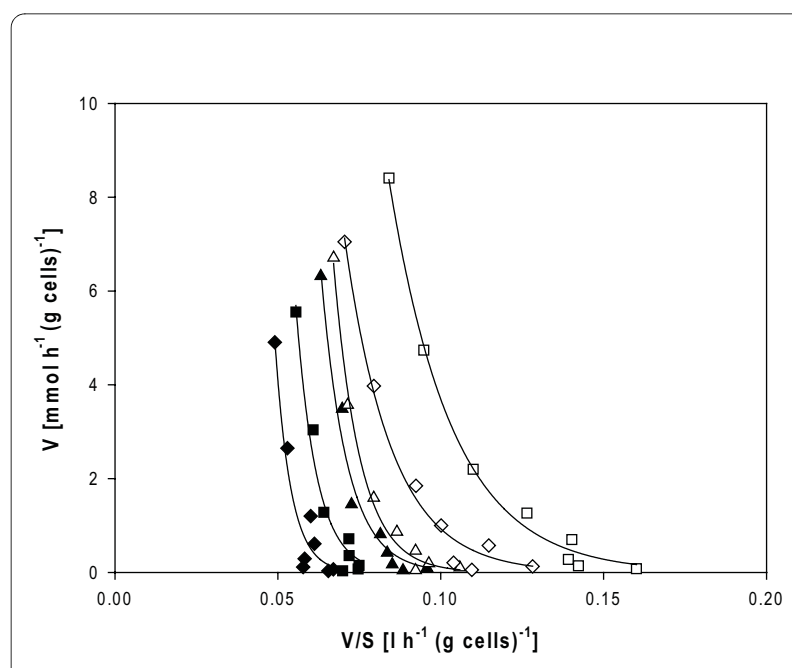

Figure $4 \mathrm{D}-\left[\mathrm{U}-{ }^{14} \mathrm{C}\right]$ xylose initial uptake rates in Saccharomyces cerevisiae strains engineered for xylose and arabinose utilization. Eadie-Hofstee plot of D-[U-14 C]xylose initial uptake rates in $\mathrm{S}$. cerevisiae cells (TMB3400 (X), triangles; TMB3061 (X+A), diamonds; TMB3130 ( $X+A$ evolved), squares) grown in D-glucose (filled symbols) or D-xylose (open symbols). 
The kinetic parameters for L-[1-14C] arabinose uptake were more difficult to evaluate because the uptake capacity was much lower for L-arabinose than for D-xylose. Therefore, only $5 \mathrm{mM}$ and $100 \mathrm{mM}$ concentrations were used in L-arabinose transport experiments with strains TMB3400, TMB3061, TMB3063 and TMB 3130.

When grown in D-glucose all strains showed L-arabinose uptake rates below $0.5 \mathrm{mmol}^{-1} \mathrm{~h}^{-1}$ (g cells) ${ }^{-1}$ using 100 $\mathrm{mM}$ L-arabinose (Figure 5) which was around 10-times lower than xylose uptake under the same conditions. The tendency for L-arabinose uptake was, however, the same as for xylose uptake on glucose-grown cells (g): TMB3061 (g) < TMB3130 (g) < TMB3400 (g) (Figure 5).

When grown in L-arabinose, TMB3061 and TMB3063 showed similar uptake capacities both for $5 \mathrm{mM}$ and 100 $\mathrm{mM}$ L-arabinose $\left(0.05 \pm 0.01\right.$ and $1.0 \pm 0.3 \mathrm{mmol}^{-1} \mathrm{~h}^{-1} \mathrm{~g}$ cells $^{-1}$, respectively; Figure 5). In contrast TMB3130 showed significant improvement in L-arabinose transport when grown in L-arabinose $(0.17 \pm 0.01$ and $2.4 \pm 0.5$ mmol.h ${ }^{-1}$.g cells ${ }^{-1}$, respectively for $5 \mathrm{mM}$ and $100 \mathrm{mM} \mathrm{L-}$ arabinose) (Figure 5), representing two- to threefold higher L-arabinose uptake compared to the parental strain TMB3061.

\section{Discussion}

In the present work, evolutionary engineering was used to improve xylose and arabinose co-utilization in a recombinant industrial $S$. cerevisiae strain carrying the genes from fungal xylose and bacterial arabinose pathways chromosomally integrated in the genome. Both the aerobic growth rate and the final biomass concentration were significantly improved in the evolved strain.

Under anaerobic conditions, the evolved strain TMB3130 displayed higher total anaerobic pentose coconsumption rate as well as the highest ethanol yield. This resulted from an improved conversion of xylose to

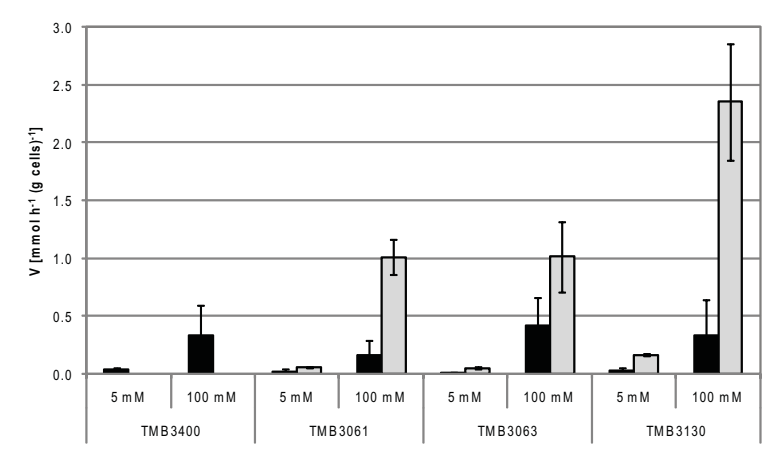

Figure 5 L-arabinose initial uptake rates in Saccharomyces cerevisiae strains engineered for xylose and arabinose utilization. L-[1${ }^{14} \mathrm{C}$ ]Arabinose initial uptake rates of S. cerevisiae cells (TMB3400 TMB3061, TMB3063 and TMB3130) grown in D-glucose (black bars) or L-arabinose (grey bars). ethanol (with less xylitol and glycerol as by-products) whereas arabinose was almost stochiometrically converted to arabitol via XR enzyme (Figure 1). We suspect that the use of XR for arabinose conversion to arabitol triggered a shortage of reduced nicotinamide adenine dinucleotide phosphate (NADPH) for the XR reaction, as observed from the increased oxidized nicotinamide adenine dinucleotide phosphate $\left(\mathrm{NADP}^{+}\right)$-dependent acetate production. Lower NADPH may also have forced XR to use NADH for the conversion of xylose to xylitol, which has been shown to lower xylitol production as a result of a more balanced co-factor utilization between XR and $\mathrm{XDH}$ enzymes [17]. In addition, the reduced available NADH would result in lower glycerol production. Overall ethanol production should increase since less carbon is being diverted to glycerol and xylitol by-products.

Molecular and physiological analyses of the evolved isolate TMB3130 revealed that improved transport capacity and initial sugar metabolism contributed to the increased co-utilization of xylose and arabinose. In contrast the molecular basis for the improvements of the haploid laboratory strain have so far not been reported [16]. Comparing isolate TMB3130 and its parental strains at the gene level did not reveal any mutation in the heterologous genes of the pentose converting enzymes. However, the parental strain contained several mutations in AraA and few in $A r a B$, as compared to the original bacterial sequence. This may indicate a high selective pressure on the first enzymes of the arabinose utilization pathway during arabinose consumption and would confirm previous indications on the pivotal role of L-arabinose isomerase in arabinose-engineered S. cerevisiae [7]. Also two silent mutations in B. subtilis AraA were shifted towards codon usage found at increased frequencies in highly expressed genes [18].

In the evolved strain TMB3130, the XR and XDH activities increased 5.5- and 3.5-fold, respectively, as compared to the parental strain TMB3061. This concomitant increase in activity associated with unchanged gene sequences suggest that the higher $\mathrm{XR}$ and $\mathrm{XDH}$ activities result from one or several duplications on the genome of the integrative vector that carry $X Y L 1$ and $X Y L 2$ genes. $\mathrm{XR}$ and $\mathrm{XDH}$ levels were also higher than for the original strain TMB3400, although xylose consumption rates were in the same range. Therefore, the loss of xylose consumption capacity in strain TMB3061 was not associated with decreased XR-XDH activity whereas the recovery of xylose consumption capacity in the evolved strain was. It suggests that $S$. cerevisiae is able to use different strategies to recover a phenotype under selection pressure.

Under glucose limitation or absence of glucose, highaffinity glucose transporters are induced [19]. This correlates with the higher pentose uptake rate that was observed when strains were pre-grown on xylose or arab- 
inose instead of glucose since hexose transporters have been shown to also transport pentoses: Hxt4p, Hxt5p, Hxt7p and Gal2p can transport xylose [20,21] whereas Gal2p can also transport arabinose [22]. Xylose and arabinose transport was improved in the evolved strain TMB3130, 1.2- to 1.5-fold and two- to threefold, respectively. This suggests an improvement in pentose uptake rate through evolution and confirms that continuous cultivation under substrate limitation tends to generate an evolved population with improvement at the level of nutrient transport $[12,13]$. Specific xylose and arabinose transporters have been reported in yeast [23] and overexpression of one or several of these transporters may further improve simultaneous pentose fermentation.

Despite significant improvement, the xylose and arabinose co-utilization rate of the evolved industrial strain TMB3130 was three- and fivefold lower, respectively, than what was achieved with the evolved laboratory strain [16]. TMB3130 was derived from TMB3400 that is at least diploid and originates from a cross between winemaking strains [24]. It has been suggested that it is more difficult to obtain substantial strain improvement by evolution of industrial S. cerevisiae strains compared to haploid laboratory strains, because of the intrinsic genetic robustness that result from the polyploidy of industrial strains $[11,14]$. This would explain the limited success of evolutionary approaches with industrial $S$. cerevisiae strains for bioethanol production $[14,25]$.

The evolutionary engineering protocol used to increase the consumption rate of xylose and arabinose in the laboratory $S$. cerevisiae strain [16] and in the currently reported industrial S. cerevisiae strain differed significantly in the selection method. The laboratory strain was selected through repeated batch cultivations in media alternating combinations of glucose, xylose and arabinose sugars, after limited success in evolving the parent under chemostat conditions [16]. In contrast, the industrial strain was improved by increasing the dilution rate in a continuous cultivation with a defined medium containing a mixture of xylose and arabinose as sole carbon sources. The industrial strain carried all genes from the heterologous pentose pathways on the genome whereas the engineered CEN.PK strain combined integrated and plasmidborn genes. On the one hand multicopy plasmids may favour fine tuning of the gene copy number to match the required expression levels, on the other hand it may be more difficult to reach a stable phenotype for mixed sugar utilization unless careful selection pressure (such as successive cultivation on alternative sugars) is applied. It has previously been reported that a strain harbouring a multicopy plasmid was more prone to evolution for improved aerobic growth on xylose than the corresponding strain with all genes integrated on the genome [13]. Therefore, one can speculate that plasmids may increase the strain instability, thereby increasing the mutation or recombination rate and favour evolution.

\section{Conclusions}

An industrial yeast strain was evolved for improved xylose and arabinose co-utilization through continuous cultivation at increasing dilution rate with xylose and arabinose as sole carbon sources. The xylose and arabinose consumption rates increased both under aerobic and anaerobic conditions. As a result, the aerobic growth rate and the final cell mass on xylose and arabinose increased. Under anaerobic conditions, the ethanol yield increased at the expense of the xylitol and glycerol yields, whereas arabinose was almost entirely converted to arabitol. Increased transport of xylose and arabinose as well as increased levels of xylose converting enzymes most likely contributed to the improvement of pentose utilization. To the best of our knowledge, this is the first report on improved mixed-pentose utilization that characterizes the molecular mechanisms for the evolutionary engineered strain.

\section{Methods}

\section{Strains}

Yeast strains used in the study are summarized in Table 1. All strains were stored in $15 \%$ glycerol at $-80^{\circ} \mathrm{C}$.

\section{Aerobic cultivations}

Aerobic cultivation was performed on YNB $\left(6.7 \mathrm{~g} \mathrm{~L}^{-1}\right.$ Difco YNB without amino acids; Becton, Dickinson and Company, MD, USA). YNB medium was supplemented with $50 \mathrm{~g} \mathrm{~L}^{-1}$ arabinose, $50 \mathrm{~g} \mathrm{~L}^{-1}$ xylose or $20 \mathrm{~g} \mathrm{~L}^{-1}$ glucose unless otherwise stated. For aerobic growth determinations, pre-cultivation conditions were normalized by growing the cells in YNB medium with $20 \mathrm{~g} \mathrm{~L}^{-1}$ glucose until late exponential phase from which cell culture was used to inoculate YNB medium with arabinose or xylose at an $\mathrm{OD}_{620 \mathrm{~nm}}$ of 0.2. Double concentration of YNB was used when the sugar concentration was above $20 \mathrm{~g} \mathrm{~L}^{-1}$. YNB medium was buffered with potassium hydrogen phthalate (10.21 $\mathrm{g} \mathrm{L}^{-1}$ phthalate, $\left.2.1 \mathrm{~g} \mathrm{~L}^{-1} \mathrm{KOH}\right)$ at $\mathrm{pH} 5.5$ [26]. The cultures were grown in cotton-stoppered baffled shake-flasks with starting medium volume of $10 \%$ of the flask volume. Temperature was controlled at $30^{\circ} \mathrm{C}$ and shaking was set at 180-200 rpm (Gallenkamp INR-200, Leicester, UK). Cell growth was monitored by determining the optical density (OD $620 \mathrm{~nm}$ ) with a Hitachi U-1800 Spectrophotometer (Hitachi Ltd, Tokyo, Japan). YPD (10 $\mathrm{g} \mathrm{L}^{-1}$ yeast extract, $20 \mathrm{~g} \mathrm{~L}^{-1}$ peptone, $20 \mathrm{~g} \mathrm{~L}^{-1}$ glucose, $20 \mathrm{~g}$ $\mathrm{L}^{-1}$ agar) or YNB $\left(6.7 \mathrm{~g} \mathrm{~L}^{-1}, 30 \mathrm{~g} \mathrm{~L}^{-1}\right.$ agar) plates were used for cell culture from frozen stocks, for inoculation and for recovery of cells from the chemostat or isolation of the evolvants. 


\section{Continuous culture for evolutionary engineering}

The pre-culture of TMB3061 cells was grown aerobically using YNB medium with $20 \mathrm{~g} \mathrm{~L}^{-1}$ glucose. The cells were harvested and mixed with the fermentation medium to an OD $620 \mathrm{~nm}$ of 0.5 . The continuous cultivation was performed in a $250 \mathrm{~mL}$ in-house-built jacketed bioreactor on defined medium [27] that contained $0.05 \%$ (vol/vol) antifoam (Dow Corning Antifoam RD Emulsion; BDH Laboratory Supplies, Dorset, UK). For the initial batch culture, $150 \mathrm{~mL}$ of working volume was used with $500 \mathrm{rpm}$ magnetic stirring and for the chemostat culture the volume was reduced to approximately $100 \mathrm{~mL}$ with $250 \mathrm{rpm}$ stirring. Air was supplied by sparging $0.8 \mathrm{~L}$ air gas $\mathrm{min}^{-1}$ controlled with a gas flow-meter (Bronkhorst, HI-TECH, Ruurlo, The Netherlands). The temperature was set at $30^{\circ} \mathrm{C}$ and $\mathrm{pH}$ was maintained at 5.5 by automatic addition of $0.3 \mathrm{M} \mathrm{KOH}$. Outlet gases from the culture were followed with a Carbon Dioxide and Oxygen Monitor type 1308 (Brüel and Kjaer, Copenhagen, Denmark).

In the initial batch culture, $10 \mathrm{~g} \mathrm{~L}^{-1}$ glucose and $10 \mathrm{~g} \mathrm{~L}^{-1}$ xylose were used as carbon sources. Then the carbon source in the feed was $10 \mathrm{~g} \mathrm{~L}^{-1}$ xylose, followed by a mixture of $5 \mathrm{~g} \mathrm{~L}^{-1}$ xylose and $5 \mathrm{~g} \mathrm{~L}^{-1}$ arabinose. The initial dilution rate $\left(0.02 \mathrm{~h}^{-1}\right)$ was increased stepwise every time the culture reached a steady state. The absence of contamination was checked by microscopic examination.

\section{Anaerobic co-fermentation of glucose and pentose}

For the characterization of evolved strains, pre-cultures were grown in defined mineral medium [27] with $20 \mathrm{~g} \mathrm{~L}^{-1}$ glucose buffered to $\mathrm{pH} 5.5$ with potassium hydrogen phthalate [26]. A first pre-culture grown overnight in a test tube was used to inoculate a second $100 \mathrm{~mL}$ pre-culture in a baffled $1 \mathrm{~L}$ flask. Cells from late exponential phase were harvested, washed twice with $0.9 \% \mathrm{NaCl}$ and inoculated at an OD $620 \mathrm{~nm}$ of 0.2. Fermentation was conducted in 3 L Applikon Bio Reactors (Applikon, Schiedam, The Netherlands) with a working volume of $1.5 \mathrm{~L}$. Defined mineral medium [27] supplemented with a mixture of $20 \mathrm{~g} \mathrm{~L}^{-1}$ glucose, $20 \mathrm{~g} \mathrm{~L}^{-1}$ xylose and $20 \mathrm{~g} \mathrm{~L}^{-1}$ arabinose was used. For anaerobic fermentation $0.4 \mathrm{~g} \mathrm{~L}^{-1}$ Tween- 80 and $0.01 \mathrm{~g} \mathrm{~L}^{-1}$ ergosterol were added to the medium. Temperature was set at $30^{\circ} \mathrm{C}$, agitation at 200 $\mathrm{rpm}$ and $\mathrm{pH}$ was maintained at 5.5 by automatic addition of $3 \mathrm{M} \mathrm{KOH}$. Anaerobic conditions were obtained by sparging nitrogen (AGA Gas, Sundbyberg, Sweden) at a flow rate of $0.2 \mathrm{~L} \mathrm{~min}{ }^{-1}$. The experiments were performed at least in duplicate.

\section{Analysis of metabolites and cell dry weight}

The concentrations of xylose, glucose, acetic acid, glycerol, xylitol and ethanol were determined in the filtered sample supernatant by high performance liquid chroma- tography (Beckman Instruments, CA, USA) using Aminex HPX-87H ion exchange columns (Bio-Rad, CA, USA) at $45^{\circ} \mathrm{C}$ with $5 \mathrm{mM} \mathrm{H}_{2} \mathrm{SO}_{4}$ as mobile phase and at a flow rate of $0.6 \mathrm{~mL} \mathrm{~min}^{-1}$. A series of three connected columns was used for separation of glucose, xylose and arabinose and their corresponding fermentation by-products when present in the same sample. Arabinose, arabitol and xylitol were separated using a HPX-87P ion exchange column (Bio-Rad, CA, USA) at $85^{\circ} \mathrm{C}$ with a mobile phase of water at a flow rate of $0.5 \mathrm{~mL} \mathrm{~min}{ }^{-1}$. A RID-6A refractive index detector (Shimadzu, Kyoto, Japan) was used for the detection of all the compounds. Ethanol concentration was calculated from the degree of reduction balance.

Cell dry weight was determined in triplicates by filtering $5 \mathrm{~mL}$ culture through a pre-weighed hydrophilic polyethersulfone filter (PALL, Life Sciences, Michigan, USA).

\section{Enzyme activities}

$\mathrm{XR}$ and $\mathrm{XDH}$ assays were performed with an Ultrospec 2100 pro spectrophotometer (Amersham Biosciences, Uppsala, Sweden). Freshly streaked cells grown in YNB plates supplemented with $20 \mathrm{~g} \mathrm{~L}^{-1}$ glucose were inoculated in tubes containing YNB liquid medium with $20 \mathrm{~g} \mathrm{~L}^{-}$ ${ }^{1}$ glucose. Overnight grown cells were then used to inoculate shake flask cultures with $20 \mathrm{~g} \mathrm{~L}^{-1}$ glucose. Cells were harvested in exponential phase and washed twice with water. Y-PER reagent (Pierce Biotechnology, IL, USA) was used to extract proteins. The protein concentration was determined with the Coomassie Plus protein assay reagent (Pierce, IL, USA) with bovine serum albumine as standard. XR activity was measured as previously described $[28,29]$. For the $\mathrm{XDH}$ assay [30] triethanolamine buffer at pH 7 [31] was used. Experiments were performed in biological triplicates and with duplicates of different concentrations of the extracted proteins. Arabinose isomerase activity could not be determined because of the unavailability of commercial reagents.

\section{Genetic analysis}

In order to verify that the isolates originated from TMB3061, the overexpressed endogenous xylulokinase gene was amplified by PCR using primers binding to the heterologous $P G K 1$ promoter and to the coding sequence of XKS1 gene. Yeast genomic DNA was extracted with Easy-DNA Kit (Invitrogen, Groningen, The Netherlands) or by phenol/chloroform method. The AraA, AraB, AraD, $X Y L 1$ and $X Y L 2$ genes were sequenced in strains TMB3061 and TMB3130. The Pfu DNA polymerase or high fidelity PCR enzyme mix that were used for PCR amplification were obtained from Fermentas (Vilnius, Lithuania). The QIAquick Gel Extraction Kit (Qiagen $\mathrm{GmbH}$, Hilden, Germany) was used for purification of DNA fragments from agarose gel. DNA sequencing and 
oligonucleotide synthesis was performed at Eurofins MWG Operon (Martinsried, Germany).

\section{Transport kinetics}

Cells were inoculated in shake flasks with medium (volume equal to one-fifth of flask capacity) containing YNB (without amino acids) and D-glucose, D-xylose or L-arabinose $\left(20 \mathrm{~g} \mathrm{~L}^{-1}\right)$. Cells were grown aerobically at $30^{\circ} \mathrm{C}, 180$ $\mathrm{rpm}$, to exponential phase $\left(\mathrm{OD}_{640} \mathrm{~nm}=1\right)$. Initial uptake rates were determined at $25^{\circ} \mathrm{C}$ [32], using $\mathrm{D}-\left[\mathrm{U}-{ }^{14} \mathrm{C}\right] \mathrm{glu}$ cose, D-[U- $\left.{ }^{14} \mathrm{C}\right]$ xylose (GE Healthcare, Buckinghamshire, UK) or L-[1-14C]arabinose (Moravek Biochemicals Inc, CA, USA). D-[U- $\left.{ }^{14} \mathrm{C}\right]$ Xylose uptake measurements were performed using a sugar concentration range between 0.5 $\mathrm{mM}$ and $100 \mathrm{mM}$ (final concentrations) and a period of $10 \mathrm{~s} . \mathrm{L}-\left[1-{ }^{14} \mathrm{C}\right]$ arabinose uptake measurements were performed using sugar concentrations of $5 \mathrm{mM}$ and $100 \mathrm{mM}$ (final concentrations) and a period of 5-10 s.

\section{Abbreviations}

OD: optical density; PCR: polymerase chain reaction; XDH: xylitol dehydrogenase; XR: xylose reductase; YNB: yeast nitrogen base; YPD: yeast peptone glucose.

\section{Competing interests}

The authors declare that they have no competing interests.

\section{Authors' contributions}

CF and VSN contributed equally. RGS participated in the experimental design, performed the physiological analysis of the mutant strains, participated in screening, fermentation experiments, initial experiments of transport assays, sequencing, enzymatic assays, data analysis and drafted the manuscript. KK participated in initial planning of the study and in the experimental design, performed chemostat cultivations and helped to draft the manuscript. CF designed and performed transport assays and commented the manuscript. VSN participated in the screening and physiological analysis of mutant strains. JRMA, OB and CUL participated in initial planning of the study and performed chemostat cultivations. MB participated in fermentations performance. $\mathrm{BHH}$ participated in initial planning of the study and in the experimental design and edited the manuscript. MFGG planned the study, participated in the experimental design and edited the manuscript
}

\section{Acknowledgements \\ We are very grateful to the late Isabel Spencer-Martins for fruitful and extraordi- nary collaboration and for discussions and guidance in designing the transport assays. \\ We thank the Swedish Energy Agency and EU project NILE (FP6-019882) for financial support.}

\footnotetext{
Author Details

1Department of Applied Microbiology, Lund University, PO Box 124, SE-22100 Lund, Sweden, ${ }^{2}$ Center for Microbial Biotechnology, Department of Systems Biology, Technical University of Denmark, Soltofts plads, 2800 Kgs Lyngby, Denmark, ${ }^{3}$ Centro de Recursos Microbiológicos (CREM), Departamento de Ciências da Vida, Faculdade de Ciências e Tecnologia, Universidade Nova de Lisboa, 2829-516 Caparica, Portugal, 4Laboratório Nacional de Energia e Geologia, I.P., Unidade de Bioenergia, Estrada do Paço do Lumiar 22, 1649-038, Lisboa, Portugal, ${ }^{5}$ Carlsberg Research Center, Gamle Carlsberg vej 10, DK-2500 Valby, Denmark and ${ }^{6}$ Department of Chemistry, Biotechnology and Food Science Norwegian University of Life Sciences, PO Box 5003, N-1432 Ås, Norway
}

Received: 10 March 2010 Accepted: 15 June 2010

Published: 15 June 2010
References

1. Hayn MSW, Klinger R, Steinmüller H, Sinner M, Esterbauer H: Basic research and pilot studies on the enzymatic conversion of lignocellulosics. In Bioconversion of Forest and Agricultural Plant Residues Edited by: Saddler JN. Wallingford: CAB International; 1993:33-72.

2. Hahn-Hägerdal B, Karhumaa K, Jeppsson M, Gorwa-Grauslund MF: Metabolic engineering for pentose utilization in Saccharomyces cerevisiae. In Biofuels Volume 108. Berlin/Heidelberg: Springer; 2007:147-177.

3. Matsushika A, Inoue H, Kodaki T, Sawayama S: Ethanol production from xylose in engineered Saccharomyces cerevisiae strains: current state and perspectives. Applied Microbiology and Biotechnology 2009, 84(1):37-53.

4. Karhumaa K, Garcia Sanchez R, Hahn-Hägerdal B, Gorwa-Grauslund MF: Comparison of the xylose reductase-xylitol dehydrogenase and the xylose isomerase pathways for xylose fermentation by recombinant Saccharomyces cerevisiae. Microbial Cell Factories 2007, 6:5.

5. Becker J, Boles E: A modified Saccharomyces cerevisiae strain that consumes L-Arabinose and produces ethanol. Appl Environ Microb 2003, 69(7):4144-4150.

6. Wisselink HW, Toirkens MJ, del Rosario Franco Berriel M, Winkler AA, van Dijken JP, Pronk JT, van Maris AJA: Engineering of Saccharomyces cerevisiae for efficient anaerobic alcoholic fermentation of L-Arabinose. Appl Environ Microb 2007, 73(15):4881-4891.

7. Wiedemann B, Boles E: Codon-optimized bacterial genes improve LArabinose fermentation in recombinant Saccharomyces cerevisiae. Appl Environ Microb 2008, 74(7):2043-2050.

8. Richard P, Verho R, Putkonen M, Londesborough J, Penttilä M: Production of ethanol from L-arabinose by Saccharomyces cerevisiae containing a fungal L-arabinose pathway. FEMS Yeast Research 2003, 3(2):185-189.

9. Bettiga M, Bengtsson O, Hahn-Hägerdal B, Gorwa-Grauslund MF: Arabinose and xylose fermentation by recombinant Saccharomyces cerevisiae expressing a fungal pentose utilization pathway. Microbial Cell Factories 2009, 8:40

10. Karhumaa K, Wiedemann B, Hahn-Hägerdal B, Boles E, Gorwa-Grauslund MF: Co-utilization of L-arabinose and D-xylose by laboratory and industrial Saccharomyces cerevisiae strains. Microbial Cell Factories 2006, 5:18.

11. Sauer $\mathrm{U}$ : Evolutionary engineering for industrially important microbial phenotypes. Advances in Biochemical Engineering/Biotechnology 2001, 73:129-169.

12. Kuyper M, Toirkens MJ, Diderich JA, Winkler AA, van Dijken JP, Pronk JT: Evolutionary engineering of mixed-sugar utilization by a xylosefermenting Saccharomyces cerevisiae strain. FEMS Yeast Research 2005, 5(10):925-934.

13. Pitkänen JP, Rintala E, Aristidou A, Ruohonen L, Penttilä M: Xylose chemostat isolates of Saccharomyces cerevisiae show altered metabolite and enzyme levels compared with xylose, glucose, and ethanol metabolism of the original strain. Applied Microbiology and Biotechnology 2005, 67(6):827-837.

14. Sonderegger M, Jeppsson M, Larsson C, Gorwa-Grauslund MF, Boles E, Olsson L, Spencer-Martins I, Hahn-Hägerdal B, Sauer U: : Fermentation performance of engineered and evolved xylose-fermenting Saccharomyces cerevisiae strains. Biotechnology and Bioengineering 2004, 87(1):90-98

15. Sonderegger M, Sauer U: Evolutionary engineering of Saccharomyces cerevisiae for anaerobic growth on xylose. Appl Environ Microb 2003, 69(4):1990-1998.

16. Wisselink HW, Toirkens MJ, Wu Q, Pronk JT, Van Maris AJA: Novel evolutionary engineering approach for accelerated utilization of glucose, xylose and arabinose mixtures by engineered Saccharomyces cerevisiae. Appl Environ Microb 2009, 75(4):907-914.

17. Jeppsson M, Johansson B, Hahn-Hägerdal B, Gorwa-Grauslund MF: Reduced oxidative pentose phosphate pathway flux in recombinant xylose-utilizing Saccharomyces cerevisiae strains improves the ethanol yield from xylose. Appl Environ Microb 2002, 68(4):1604-1609.

18. Sharp PM, Cowe E: Synonymous codon usage in Saccharomyces cerevisiae. Yeast 1991, 7:657-678.

19. Kruckeberg AL: The hexose transporter family of Saccharomyces cerevisiae. Arch Microbiol 1996, 166(5):283-292.

20. Hamacher T, Becker J, Gárdonyi M, Hahn-Hägerdal B, Boles E: Characterization of the xylose-transporting properties of yeast hexose 
transporters and their influence on xylose utilization. Microbiology 2002, 148(Pt 9):2783-2788.

21. Sedlak M, Ho NWY: Characterization of the effectiveness of hexose transporters for transporting xylose during glucose and xylose cofermentation by a recombinant Saccharomyces yeast. Yeast 2004, 21(8):671-684.

22. Cirillo VP: Galactose transport in Saccharomyces cerevisiae. I. nonmetabolized sugars as substrates and inducers of galactose transport system. J Bacteriol 1968, 95(5):1727-1731.

23. Leandro MJ, Fonseca C, Gonçalves P: Hexose and pentose transport in ascomycetous yeasts: an overview. FEMS Yeast Research 2009, 9(4):511-525. fingerprinting and karyotyping i

24. Vander Westhuizen TJ, Pretorius IS: The value of electrophoretic fingerprinting and karyotyping in wine yeast breeding programs. Antonie Van Leeuwenhoek Int J Gen Molec Microbiol 1992, 61(4):249-257.

25. Heer D, Sauer U: Identification of furfural as a key toxin in lignocellulosic hydrolysates and evolution of a tolerant yeast strain. Microbial Biotechnol 2008, 1(6):497-506.

26. Hahn-Hägerdal B, Karhumaa K, Larsson CU, Gorwa-Grauslund M, Görgens J, van Zyl WH: Role of cultivation media in the development of yeast strains for large scale industrial use. Microbial Cell Factories 2005, 4:31.

27. Jeppsson M, Bengtsson O, Franke K, Lee H, Hahn-Hägerdal B, GorwaGrauslund MF: The expression of a Pichia stipitis xylose reductase mutant with higher K(M) for NADPH increases ethanol production from xylose in recombinant Saccharomyces cerevisiae. Biotechnology and Bioengineering 2006, 93(4):665-673.

28. Eliasson A, Christensson C, Wahlbom CF, Hahn-Hägerdal B: Anaerobic xylose fermentation by recombinant Saccharomyces cerevisiae carrying $X Y L 1, X Y L 2$, and $X K S 1$ in mineral medium chemostat cultures. Appl Environ Microb 2000, 66(8):3381-3386.

29. Smiley KL, Bolen PL: Demonstration of D-xylose reductase and D-xylitol dehydrogenase in Pachysolen tannophilus. Biotech Lett 1982, 4:607-610.

30. Rizzi MHK, Erlemann P, Bui-Thahn NA, Dellweg H: Purification and properties of the $\mathrm{NAD}^{+}$-xylitol-dehydrogenase from the yeast Pichia stipitis. J Fermentation Bioengineering 1989, 67:20-24.

31. Wahlbom CF, van Zyl WH, Jönsson LJ, Hahn-Hägerdal B, Otero RR: Generation of the improved recombinant xylose-utilizing Saccharomyces cerevisiae TMB 3400 by random mutagenesis and physiological comparison with Pichia stipitis CBS 6054. FEMS Yeast Res 2003, 3(3):319-326.

32. Fonseca C, Romão R, Rodrigues de Sousa H, Hahn-Hägerdal B, SpencerMartins I: L-Arabinose transport and catabolism in yeast. FEBS J 2007, 274(14):3589-3600.

doi: 10.1186/1754-6834-3-13

Cite this article as: Garcia Sanchez et al., Improved xylose and arabinose utilization by an industrial recombinant Saccharomyces cerevisiae strain using evolutionary engineering Biotechnology for Biofuels 2010, 3:13

Submit your next manuscript to BioMed Central and take full advantage of:

- Convenient online submission

- Thorough peer review

- No space constraints or color figure charges

- Immediate publication on acceptance

- Inclusion in PubMed, CAS, Scopus and Google Scholar

- Research which is freely available for redistribution

Submit your manuscript at www.biomedcentral.com/submit
C BioMed Central 